\title{
Stochastic Analysis of Unsaturated Flow Subject to Temporally Correlated Infiltration
}

\author{
Ching-Min Chang • Hund-Der Yeh
}

Received: 16 May 2013 / Accepted: 12 May 2014 / Published online: 27 May 2014

C) Springer Science+Business Media Dordrecht 2014

\begin{abstract}
In this article, we attempt to quantify the variability of water pressure head in response to temporally correlated fluctuations in infiltration rate in partially saturated heterogeneous formations. This study concentrates on the unsaturated case of predominantly vertical movement of moisture. A closed-form solution is developed to analyze the impacts of the temporal correlation scale of infiltration fluctuations and the spatial correlation scale of $\log$-saturated hydraulic conductivity $\left(\ln K_{S}\right)$ field on the variability in water pressure head. Our result indicates that the temporal correlation scale of infiltration process or the spatial correlation scale of $\ln K_{S}$ field takes a role in increasing the variability in the pressure head.
\end{abstract}

Keywords Stochastic analysis · Unsaturated flow · Water pressure head variance

\section{Introduction}

Surface water from various sources moves downward through the unsaturated zone to the water table. The understanding of the movement of soil moisture in the unsaturated zone is essential to determine the replenishment of phreatic aquifers. In addition, contaminants from sources at ground surface travel down with the infiltrating water towards the water table. The prediction of the movement of moisture in the unsaturated zone is needed if the subsurface remediation is necessary.

It is well known that the movement of unsaturated flow is affected profoundly by the existence of natural variability of hydrological phenomena, such as temporal fluctuations in infiltration rate or spatial variation in hydraulic conductivity. Since the detailed description of the complex natural phenomena is impossible, a possible approach is to represent the natural heterogeneity in terms of random processes characterized by a finite number of statistical

C.-M. Chang · H.-D. Yeh $(\varangle)$

Institute of Environmental Engineering, National Chiao Tung University,

1001 University Road, Hsinchu 300, Taiwan, Republic of China

e-mail: hdyeh@mail.nctu.edu.tw 
parameters (e. g., Mantoglou and Gelhar 1987; Mantoglou 1992; Russo 1995; Foussereau et al. 2000; Lu and Zhang 2002; Zhang and Lu 2002; Li et al. 2009). As such, the stochastic approach leads to solutions for describing the field-scale mean flow behavior in terms of effective hydraulic conductivity and quantifying the variability of pressure head in response to natural heterogeneity in terms of pressure head variance. Comprehensive overviews of the construction of the stochastic approach and its application for modeling the field-scale flow processes in heterogeneous media may refer to Dagan (1989), Gelhar (1993), Zhang (2002), and Rubin (2003).

It has been recognized that the introduction of infiltration impacts the head gradient (e.g., Yoo et al. 1998; Li and Graham 1999; Settin et al. 2007). In other words, the infiltration forcing plays a key role influencing the soil moisture dynamics. Infiltration events exhibit considerable variability on temporal scales (e.g., Veneziano and Iacobellis 2002; Rodriguez-Iturbe et al. 2006; Rupp et al. 2009). Their heterogeneities can significantly affect groundwater movement in soils. As expected, there will be a great deal of uncertainty anticipated in applying the classical unsaturated flow model. Motivated by that, quantification of the uncertainty associated with the conversion of infiltration into the groundwater flow process is the focus of this study.

Existing field observations of unsaturated flow are very limited because of the complexity of the problem. Natural heterogeneity plays an important role in the field-scale unsaturated flow process. Laboratory (i.e., small or local scale) models cannot realistically produce the natural heterogeneity exhibited in the field. It is, therefore, difficult to draw conclusions from small-scale models for the processes occurring in the field. In order to produce realistic results for field applications, the development of field-scale unsaturated flow model and the quantification of its variability are indeed needed. Motivated by that, this study is, therefore, devoted to providing an analytical basis for quantifying the variability of field-scale unsaturated flow processes in response to temporally correlated fluctuations in infiltration rate and the spatial variability of soil properties. Although with some simplified assumptions, to the best of our knowledge, the closed-form expression of the head variance for the field-scale temporal vertical movement of soil moisture has so far not been reported regarding the application of the perturbation-based nonstationary spectral techniques.

The stochastic approach based on the representation of natural heterogeneity as a random field will lead to a simple head variance relationship in terms of statistical properties of input parameters. The approach thereby provides a basis for analyzing the influence of those parameters on the field-scale unsaturated flow processes. In addition, the closed-form expression for the variance of head, as developed here, could serve as a calibration target when applying the traditional model to field situations. It may be more reasonable to present the conclusions, say, for the head solution of traditional model with one standard deviation (square root of variance) representing the uncertainty of the head solution.

In this article, we concentrate on the case where the vertical movement of the soil moisture in response to temporally correlated fluctuations in infiltration rate is dominant in the vadose zone. The unsaturated flow domain is in a formation of infinitely horizontal extent, bounded above at a certain depth below the soil surface and below by prescribed pressure heads. The initial head in the flow domain is assumed constant. To simplify the analysis, the variabilities of the initial and boundary conditions are neglected (i.e., they are treated as deterministic). Perturbation-based nonstationary spectral techniques and the superposition principle will be adopted to develop the closed-form solution for the head variance. Based on the closedform expression, the impact of statistical properties of input aquifer parameters on the head variability will also be assessed. 


\section{Stochastic Formulation}

The governing equation of unsaturated flow at the local scale may be expressed as (e.g., Zhang 2002)

$$
C \frac{\partial \psi}{\partial t}-\frac{\partial}{\partial X_{i}}\left[K(\psi) \frac{\partial \psi}{\partial X_{i}}\right]-\frac{\partial K(\psi)}{\partial X_{1}}=q(t) \quad i=1,2,3,
$$

where $C=\mathrm{d} \theta / \mathrm{d} \psi$ is the specific moisture capacity (e. g., Mantoglou and Gelhar 1987; Gelhar 1993; Zhang 2002), $\theta$ is the moisture content, $\psi$ is the water pressure head, $q$ is the transient infiltration rate representing as a source term, $K(\psi)$ is the unsaturated hydraulic conductivity, and $X_{1}$ designates the vertical coordinate measured upward. Note that the dependence of the unsaturated hydraulic conductivity and the moisture content on the fluid pressure leads to strong nonlinearity in flow processes. Furthermore, the parametric description of $K-\psi$ relationship involves empirical parameters. In this work, we parameterize the functional form for $K(\psi)$ by (Gardner 1958)

$$
K(\psi)=K_{S} \exp [\alpha \psi],
$$

where $\alpha$ is a parameter associated with soil pore-size distribution and $K_{s}$ is the saturated hydraulic conductivity.

The local soil properties (namely $\ln K_{S}$ and $C$ ) and $\psi$ are considered as random fields. The source $q$ is considered to represent the temporally correlated zero-mean fluctuations in infiltration rate. To simplify the analysis of field-scale unsaturated processes, the variability in Gardner's parameter $\alpha$ is neglected and replaced by its mean. As such, Eq. (2.1) may then be viewed as a stochastic differential equation with parameters described by random processes, and therefore a resulting stochastic output $\psi$.

The random processes $C, \ln K_{S}$ and $\psi$ are expressed as

$$
\begin{aligned}
C & =\mathrm{E}[\mathrm{C}]+\mathrm{C}^{\prime}=\Gamma+C^{\prime} \\
\ln K_{S} & =\mathrm{E}\left[\ln K_{S}\right]+f=F+f \\
\psi & =\mathrm{E}[\psi]+h=H+h
\end{aligned}
$$

in the manner that $\mathrm{E}\left[C^{\prime}\right]=0, \mathrm{E}[f]=0$, and $\mathrm{E}[h]=0$, where $\mathrm{E}[-]$ denotes the expected value. Following the approach of Gelhar (1993), after introducing the random field decompositions (Eqs. (2.3a)-(2.3c)) into Eq. (2.1) and subtracting the resulting mean equation from Eq. (2.1), one gets the first-order equation describing the fluctuations in pressure head

$$
\frac{\Gamma}{K_{M}} \frac{\partial h}{\partial t}+\frac{C^{\prime}}{K_{M}} \frac{\partial H}{\partial t}-\frac{\Gamma}{K_{M}}(\alpha h+f) \frac{\partial H}{\partial t}=\frac{\partial^{2} h}{\partial X_{i}^{2}}+\alpha\left(J_{i}+\frac{\partial H}{\partial X_{i}}\right) \frac{\partial h}{\partial X_{i}}+J_{i} \frac{\partial f}{\partial X_{i}}+\frac{q(t)}{K_{M}},
$$

where $J_{i}=\partial\left(H+X_{1}\right) / \partial X_{i}$ and $\ln K_{M}$ is simply the expected value of the unsaturated $\log$ conductivity (Gelhar 1993).

Note that the validity of the small perturbation approximation (i.e., the convergence of Eqs. (2.4) and (2.5)) is preserved by that $\sigma_{f}^{2}$ (the variance of log-saturated hydraulic conductivity) should be small compared to unity (Gutjahr and Gelhar 1981). However, the study of Monte Carlo simulations of two-dimensional flow through heterogeneous formations by Zhang and Winter (1999) confirmed the accuracy of the head moment solutions obtained from the application of the small perturbation approximation in $\sigma_{f}^{2}$ at the variance up to 4.38. Similar comparison with Monte Carlo simulations presented in Guadagnini and Neuman 
(1999) yields accurate results (namely, the statistics of hydraulic head) even for $\sigma_{f}^{2}$ as high as 4 to 5 (strongly heterogeneous media).

It is worth noting that a new numerical approach, Karhunen-Loe've-based moment equation (KLME) technique, has been proposed to solve groundwater-related problems in heterogeneous media (Zhang and Lu 2004; Liu et al. 2007; Li and Zhang 2007; Li et al. 2009; Chen et al. 2013). The KLME approach combines Karhunen-Loe've (KL) expansion of a medium property and orthogonal polynomial decompositions of dependent variables. In the KLKF method, the covariance of the medium property is efficiently approximated by a small set of eigenvalues and eigenfunctions attributed to the mean square convergence of the KL decomposition. The KLME approach is able to accurately estimate the statistical moments of the dependent variable and provides considerable computational advantages as compared with the classical Monte Carlo simulations.

It is apparent that the solution of Eq. (2.4) is dependent of the solution of the mean-head equation. In other words, the perturbation and mean equations should be treated simultaneously. However, in many practical applications, the mean pressure head is generally regarded to be slowly varying in time and space in relation to the scale of variation associated with the perturbation equation (Gelhar 1993). We may, therefore, regard the coefficients involving the mean-head solution in Eq. (2.4), such as $K_{M}, J_{i}$, and $\partial H / \partial X_{i}$, to be constant over spatial scales corresponding to the perturbations when solving Eq. (2.4). In addition, the mean-head time derivative will be very small in view of the slowly varying mean unsaturated flow (Gelhar 1993) so that the products of the perturbation terms (namely $C^{\prime}, h$, and $f$ ) and $\partial H / \partial t$ in Eq. (2.4) are negligible. As a consequence, the decoupling of the head-perturbation equation from the mean-head equation can be accomplished under the condition of slowly varying mean unsaturated flow. With this framework, Eq. (2.4) is simplified to

$$
\frac{\Gamma}{K_{M}} \frac{\partial h}{\partial t}=\frac{\partial^{2} h}{\partial X_{i}^{2}}+\alpha\left(J_{i}+\frac{\partial H}{\partial X_{i}}\right) \frac{\partial h}{\partial X_{i}}+J_{i} \frac{\partial f}{\partial X_{i}}+\frac{q(t)}{K_{M}} .
$$

Consider the case where the vertical movement of the soil moisture is dominant in the vadose zone. The assumption of one-dimensional vertical flow reduces Eq. (2.5) considerable to

$$
\frac{\Gamma}{K_{M}} \frac{\partial h}{\partial t}=\frac{\partial^{2} h}{\partial X^{2}}+\alpha(2 J-1) \frac{\partial h}{\partial X}+J \frac{\partial f}{\partial X}+\frac{q(t)}{K_{M}},
$$

where $J=J_{1}$ and $X=X_{1}$. We will seek an analytic solution of Eq. (2.6) subject to deterministic boundary and initial conditions, which imply zero perturbed boundary and initial conditions, i.e.,

$$
\begin{aligned}
h_{q}(0, t) & =0 \\
h_{q}(L, t) & =0 \\
h_{q}(X, 0) & =0 .
\end{aligned}
$$

These will provide a basis to develop an analytical solution in quantifying the variability in the pressure head.

\section{Spectral Solution}

Here, we proceed with developing the perturbation solution of Eq. (2.6) and then characterizing the pressure head variation around the mean based on it. These will be performed by applying the superposition principle and the Fourier-Stieltjes representation. 
Note that Eq. (2.6) describes the fluctuations in pressure head field in response to two independent forcing terms, the last two terms on the right-hand side of Eq. (2.6), due to the spatial variability of soil properties and the temporal variability of infiltration rate. Recognition of each of the forcing terms in Eq. (2.6) presented in a linear manner, the principle of superposition is, therefore, allowed to solve Eq. (2.6) by decomposing it into two subequations according to the forcing terms, as

$$
\frac{\Gamma}{K_{M}} \frac{\partial h_{q}}{\partial t}=\frac{\partial^{2} h_{q}}{\partial X^{2}}+\alpha(2 J-1) \frac{\partial h_{q}}{\partial X}+\frac{q(t)}{K_{M}}
$$

and

$$
\frac{\Gamma}{K_{M}} \frac{\partial h_{f}}{\partial t}=\frac{\partial^{2} h_{f}}{\partial X^{2}}+\alpha(2 J-1) \frac{\partial h_{f}}{\partial X}+J \frac{\partial f}{\partial X} .
$$

We then find the response to each subpart at a time, and add the individual responses to obtain the solution of the original equation,

$$
h=h_{q}+h_{f} .
$$

Consequently, the pressure head variance to the original problem is the sum of variances of $h_{q}$ and $h_{f}$.

Considering the stochastic processes of $q$ and $f$ to be second-order stationary and using the Fourier-Stieltjes representation, both can be defined with respect to time and space, respectively, as

$$
q(t)=\int_{-\infty}^{\infty} \exp [i \omega t] \mathrm{d} Z_{q}(\omega)
$$

and

$$
f(X)=\int_{-\infty}^{\infty} \exp [i R X] \mathrm{d} Z_{f}(R)
$$

where $\omega$ is the frequency; $R$ is the wave number; and $\mathrm{d} Z_{q}$ and $\mathrm{d} Z_{f}$ are the Fourier amplitudes of the fluctuations $q$ and $f$, respectively. The responses to two forcing terms in Eqs. (3.1) and (3.2) may, therefore, be expressed, respectively, as (e.g., Li and McLaughlin 1991)

$$
h_{q}(X, t)=\int_{-\infty}^{\infty} \Phi_{q}(X, t, \omega) \mathrm{d} Z_{q}(\omega)
$$

and

$$
h_{f}(X, t)=\int_{-\infty}^{\infty} \Phi_{f}(X, t, R) \mathrm{d} Z_{f}(R)
$$

where $\Phi_{q}$ and $\Phi_{f}$ are the unknown transfer functions. Substituting Eqs. (3.4) and (3.6) into Eq. (3.1) gives

$$
\frac{\Gamma}{K_{M}} \frac{\partial \Phi_{q}}{\partial t}=\frac{\partial^{2} \Phi_{q}}{\partial X^{2}}+\alpha(2 J-1) \frac{\partial \Phi_{q}}{\partial X}+\frac{\exp (i \omega t)}{K_{M}} .
$$


The analytical solution to Eq. (3.8) associated with the following boundary and initial conditions

$$
\begin{aligned}
\Phi_{q}(0, t, \omega) & =0 \\
\Phi_{q}(L, t, \omega) & =0 \\
\Phi_{q}(X, 0, \omega) & =0
\end{aligned}
$$

is in the form

$$
\Phi_{q}=\frac{2 \pi}{L^{2}} \exp (-\varepsilon X) \sum_{n=1}^{\infty} n \frac{1-\exp (0.5 \varepsilon L) \cos (n \pi)}{v} \sin \left(\frac{n \pi X}{L}\right) \frac{\exp (i \omega t)-\exp \left(-\frac{v K_{M}}{\Gamma} t\right)}{K_{M} v+i \Gamma \omega}
$$

where $\varepsilon=(2 J-1) \alpha$ and $v=\left(n^{2} \pi^{2} / L^{2}\right)+\varepsilon 2 / 4$. In the case of $K_{M} t /\left(L^{2} \Gamma\right)>>1$, the summation in Eq. (3.10) converges rapidly (e.g., Haberman 1998) and is, therefore, approximated as

$$
\Phi_{q}=\frac{2 \pi}{L^{2}} \frac{K_{M}}{\Gamma^{2}} \frac{1+\exp (0.5 \varepsilon L)}{\mu} \exp (-0.5 \varepsilon X) \sin (\pi \xi) \frac{\exp (i \omega t)-\exp (-\mu t)}{\mu+i \omega},
$$

where $\xi=X / L$ and $\mu=K_{M}\left[\left(\pi^{2} / L^{2}\right)+\varepsilon^{2} / 4\right] / \Gamma$. Then, from Eqs. (3.6) and (3.11), $h_{q}$ takes the form

$$
h_{q}(X, t)=\frac{2 \pi}{L^{2}} \frac{K_{M}}{\Gamma^{2}} \frac{1+\exp (0.5 \varepsilon L)}{\mu} \exp (-0.5 \varepsilon X) \sin (\pi \xi) \int_{-\infty}^{\infty} \frac{\exp (i \omega t)-\exp (-\mu t)}{\mu+i \omega} d Z_{q}(\omega) .
$$

Using the representation theorem leads Eq. (3.12) to

$$
\begin{aligned}
\sigma_{h_{q}}^{2}= & \mathrm{E}\left[h_{q} h_{q}^{*}\right]=\frac{4 \pi^{2}}{L^{4}} \frac{K_{M}^{2}}{\Gamma^{2}}\left[\frac{1+\exp (0.5 \varepsilon L)}{\mu}\right]^{2} \exp (-\varepsilon X) \sin ^{2}(\pi \xi) \\
& \times \int_{-\infty}^{\infty} \frac{1-2 \cos (\omega t) \exp (-\mu t)+\exp (-2 \mu t)}{\mu^{2}+\omega^{2}} S_{q q}(\omega) d \omega,
\end{aligned}
$$

where $\sigma_{h_{q}}^{2}$ is the variance of $h_{q}$ (the transient component of head variance), the asterisk stands for the operation of complex conjugation, and $S_{q q}(\omega)$ is the spectrum of infiltration rate perturbations.

On the other hand, the introduction of Eqs. (3.5) and (3.7) to Eq. (3.2) results in

$$
\frac{\Gamma}{K_{M}} \frac{\partial \Phi_{f}}{\partial t}=\frac{\partial^{2} \Phi_{f}}{\partial X^{2}}+\alpha(2 J-1) \frac{\partial \Phi_{f}}{\partial X}+i J R \exp (i R X)
$$

The forcing term, the last term on the right-hand side of Eq. (3.14), is independent of time. Therefore, it is reasonable to expect that the transfer function $\Phi_{f}$ becomes time invariant after sufficient time has elapsed since the initial condition. With a negligible time-derivative term, Eq. (3.14) associated with zero perturbed boundary conditions admits the following solution:

$$
\Phi_{f}=\frac{J}{\varepsilon+i R}\left[\frac{\exp (-\varepsilon X)-\exp (-\varepsilon L)}{1-\exp (-\varepsilon L)}-\exp (i R X)+\frac{1-\exp (-\varepsilon X)}{1-\exp (-\varepsilon L)} \exp (i R L)\right] .
$$


Then, it follows from Eqs. (3.7) and (3.15) that the head perturbation can be written as

$$
\begin{aligned}
h_{f}(X)= & J \int_{-\infty}^{\infty}\left[\frac{\exp (-\varepsilon X)-\exp (-\varepsilon L)}{1-\exp (-\varepsilon L)}-\exp (i R X)\right. \\
& \left.+\frac{1-\exp (-\varepsilon X)}{1-\exp (-\varepsilon L)} \exp (i R L)\right] \frac{1}{\varepsilon+i R} d Z_{f}(R) .
\end{aligned}
$$

Equation (3.16) implies from applying the representation theorem that

$$
\begin{aligned}
\sigma_{h_{f}}^{2}(X)= & J^{2} \int_{-\infty}^{\infty} \frac{1}{\varepsilon^{2}+R^{2}}\left\{1+\left[\frac{\exp (-\varepsilon X)-\exp (-\varepsilon L)}{1-\exp (-\varepsilon L)}\right]^{2}+\left[\frac{1-\exp (-\varepsilon X)}{1-\exp (-\varepsilon L)}\right]^{2}\right. \\
& -2 \frac{\exp (-\varepsilon X)-\exp (-\varepsilon L)}{1-\exp (-\varepsilon L)} \cos (R X) \\
& +2 \frac{\exp (-\varepsilon X)-\exp (-\varepsilon L)}{1-\exp (-\varepsilon L)} \frac{1-\exp (-\varepsilon X)}{1-\exp (-\varepsilon L)} \cos (R L) \\
& \left.-2 \frac{1-\exp (-\varepsilon X)}{1-\exp (-\varepsilon L)} \cos [R(L-X)]\right\} S_{f f}(R) d R
\end{aligned}
$$

where $\sigma_{h_{f}}^{2}$ is the variance of $h_{f}$ (the steady component of pressure head variance) and $S_{f f}(R)$ is the spectrum of $\ln K_{s}$.

In conclusion, the results of each part, Eqs (3.13) and (3.17), are summed to obtain the general solution of pressure head variance to the original problem.

\section{Pressure Head Variance}

The computation of the variance of $h_{q}$ requires the expression of the spectrum of the infiltration rate fluctuations. In this study, we consider that the spectrum of temporally correlated infiltration fluctuations takes the following form (Gelhar 1993; Zhang and Li 2006):

$$
S_{q q}(\omega)=\frac{\sigma_{q}^{2} \eta}{\pi\left(1+\eta^{2} \omega^{2}\right)},
$$

where $\sigma_{q}^{2}$ is the variance and $\eta$ is the temporal correlation scale of the infiltration process, respectively. The time (space) interval for which the correlation coefficient drops to an insignificant magnitude is referred to as the temporal (spatial) correction scale (e.g., Dagan 1989; Gelhar 1993; Rubin 2003). When the correction scale is larger, the data profile is anticipated to be smoother, and vice versa.

With Eq. (4.1), the integration of Eq. (3.13) over the frequency domain produces the variance of $h_{q}$ as

$$
\begin{aligned}
\sigma_{h_{q}}^{2}= & 4 \pi^{2} \sigma_{q}^{2} \frac{K_{M}^{2} \eta^{2}}{\Gamma^{4} L^{4} \mu^{2}}[1+\exp (0.5 \varepsilon L)]^{2} \exp (-\varepsilon X) \sin ^{2}(\pi \xi) \\
& {\left[\frac{1+\exp (-2 \mu t)}{\mu \eta(1+\mu \eta)}-2 \exp (-\mu t) \frac{\exp (-\mu t)-\mu \eta \exp (-t / \eta)}{\mu \eta\left(1-\mu^{2} \eta^{2}\right)}\right] . }
\end{aligned}
$$


On the other hand, the following wave number spectrum

$$
S_{f f}(R)=\frac{2 \sigma_{f}^{2} \lambda^{3} R^{2}}{\pi\left(1+\lambda^{2} R^{2}\right)^{2}}
$$

associated with a hole-type exponential correlation function of $\ln K_{s}$ field (Bakr et al. 1978) is considered to compute the variance of $h_{f}$. In conjunction with Eq. (4.3), Eq. (3.17) implies that

$$
\begin{aligned}
\sigma_{h_{f}}^{2}(X)= & \sigma_{f}^{2} J^{2} \lambda^{2}\left\{\left[1+\left(\frac{\exp (-\varepsilon X)-\exp (-\varepsilon L)}{1-\exp (-\varepsilon L)}\right)^{2}+\left(\frac{1-\exp (-\varepsilon X)}{1-\exp (-\varepsilon L)}\right)^{2}\right] \frac{1}{(1+\varepsilon \lambda)^{2}}\right. \\
& +2 \frac{\exp (-\varepsilon X)-\exp (-\varepsilon L)}{1-\exp (-\varepsilon L)} \frac{1-\exp (-\varepsilon X)}{1-\exp (-\varepsilon L)} \frac{\Theta(L)}{\left(1-\varepsilon^{2} \lambda^{2}\right)^{2}} \\
& \left.-2 \frac{\exp (-\varepsilon X)-\exp (-\varepsilon L)}{1-\exp (-\varepsilon L)} \frac{\Theta(X)}{\left(1-\varepsilon^{2} \lambda^{2}\right)^{2}}-2 \frac{1-\exp (-\varepsilon X)}{1-\exp (-\varepsilon L)} \frac{\Theta(L-X)}{\left(1-\varepsilon^{2} \lambda^{2}\right)^{2}}\right\},
\end{aligned}
$$

where

$$
\Theta(Y)=-2 \varepsilon \lambda \exp (-Y \varepsilon)+\exp (-Y / \lambda)\left[\frac{Y}{\lambda}+1-Y \varepsilon^{2} \lambda+\varepsilon^{2} \lambda^{2}\right]
$$

Now, we are in a position to calculate the closed-form expression for pressure head variance according to

$$
\sigma_{h}^{2}=\sigma_{h_{q}}^{2}+\sigma_{h_{f}}^{2},
$$

where $\sigma_{h_{q}}^{2}$, the transient component of pressure head variance, is defined in Eq. (4.2) and $\sigma_{h_{f}}^{2}$, the steady component of the pressure head variance, is defined in Eq. (4.4).

The graphical presentation of the dependence of the transient component of pressure head variance upon the temporal correlation scale of infiltration field is illustrated in Fig. 1 based on Eq. (4.2). As indicated in the figure, the temporal correlation scale has positive influence

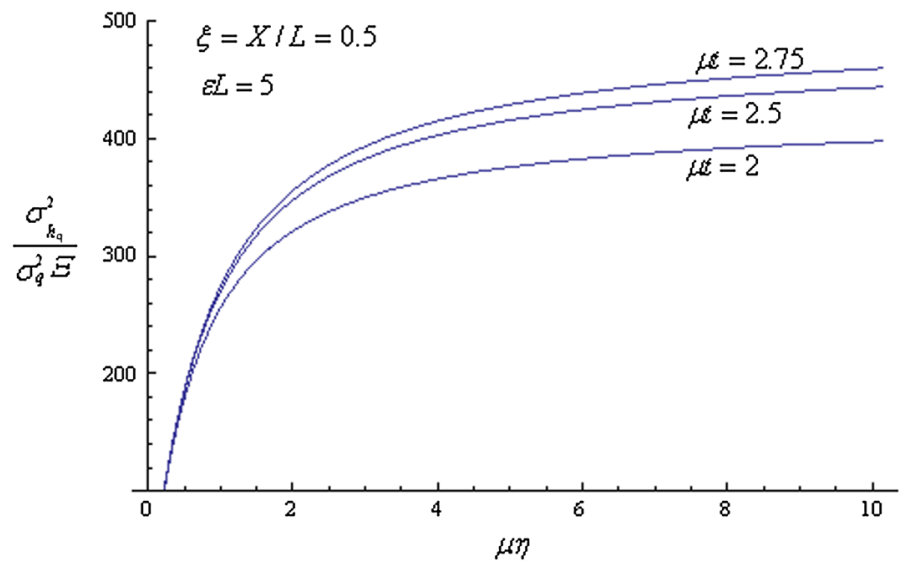

Fig. 1 Dimensionless transient component of the pressure head variance as a function of temporal correlation scale of the infiltration process, where $\Xi=\frac{1}{K_{M}^{2}\left(\frac{\varepsilon^{2}}{4}+\frac{\pi}{L}\right)^{4}}$ 


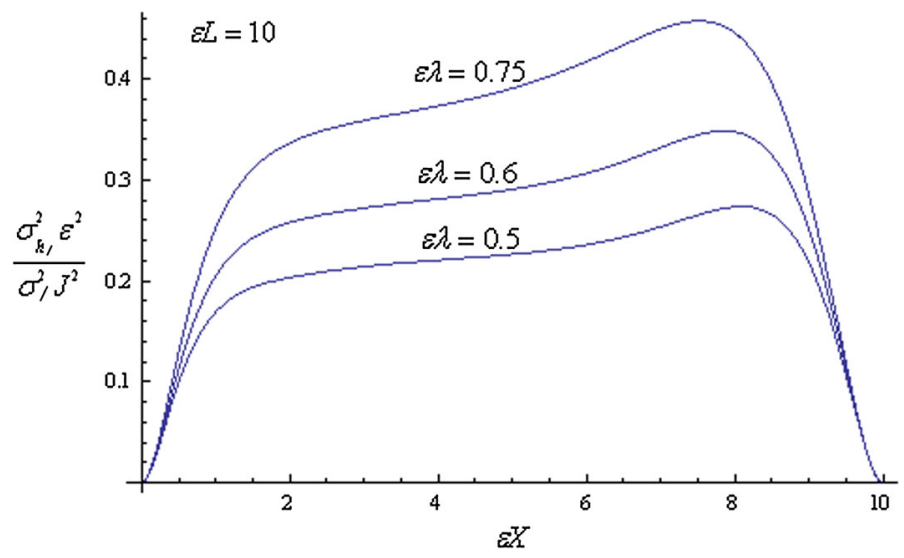

Fig. 2 Dimensionless steady component of the pressure head variance as a function of dimensionless distance from the bottom boundary

on the variation of pressure head. This is because a larger correlation scale introduces a larger temporal consistency of fluctuations in the infiltration rate, and therefore a larger temporal consistency of fluctuations in pressure head above or below the mean pressure head.

On the other hand, the plot of Eq. (4.4) as the function of position is demonstrated in Fig. 2. It indicates that for a fixed value of $\varepsilon$, the steady component of variability in pressure head created by the spatial variability in soil properties is enhanced by the spatial correlation scale of random log-saturated hydraulic conductivity $\left(\ln K_{s}\right)$ processes at a given location. As the value of spatial correlation scale is increased, the $\ln K_{s}$ processes display more persistence, having positively correlated increments. That is, positive increments tend to be followed by other positive increments, while negative increments tend to be followed by other negative increments. The profile of the $\ln K_{s}$ process is rough (i.e., more fluctuations in the $\ln K_{s}$ around the mean), and therefore produces a higher variance of pressure.

\section{Conclusions}

This article presents a stochastic analysis of transient vertical movement of soil moisture through a bounded, partially saturated heterogeneous porous medium subject to temporally correlated fluctuations in infiltration process. The use of perturbation-based nonstationary spectral perturbation techniques and the superposition principle leads to analytical solution for the variance of water pressure head, which is used to analyze the effects of the temporal correlation scale of infiltration process and the spatial correlation scale of the $\ln K_{s}$ processes. It is found that the correlation scales of infiltration and $\ln K_{s}$ processes are crucial to enhance the variability in water pressure head. As the correlation scale increases, the persistence of correlation increases and the contributions of the smallest scales of variation decrease. This implies that the fluctuations in head spend less time around their mean value of zero. As such, a larger correlation scale introduces a higher variability in head.

Acknowledgments This research is supported by the Ministry of Science under the grants NSC 101-2221E-009-105-MY2, 102-2221-E-009-072-MY2 and NSC 102-2218-E-009-013-MY3. We are grateful to the anonymous reviewers for constructive comments that improved the quality of the work. 


\section{References}

Bakr, A.A., Gelhar, L.W., Gutjahr, A.L., MacMillan, J.R.: Stochastic analysis of spatial variability in subsurface flows: 1. Comparison of one- and three-dimensional flows. Water Resour. Res. 14(2), 263-271 (1978)

Chen, X., Ng, B., Sun, Y., Tong, C.: A flexible uncertainty quantification method for linearly coupled multiphysics systems. J. Comput. Phys. 248, 383-401 (2013)

Dagan, G.: Flow and Transport in Porous Formations. Springer, New York (1989)

Foussereau, X., Graham, W., Rao, P.: Stochastic analysis of transient flow in unsaturated heterogeneous soils. Water Resour. Res. 36(4), 891-910 (2000)

Gardner, W.R.: Some steady state solutions of unsaturated moisture flow equations with application to evaporation from a water table. Soil Sci. 85(4), 228-232 (1958)

Gelhar, L.W.: Stochastic Subsurface Hydrology. Prentice Hall, Englewood Cliffs (1993)

Guadagnini, A., Neuman, S.P.: Nonlocal and localized analyses of conditional mean steady state flow in bounded, randomly nonuniform domains: 2. Computational examples. Water Resour. Res. 35(10), 3019-3039 (1999)

Gutjahr, A.L., Gelhar, L.W.: Stochastic models of subsurface flow: infinite versus finite domains and stationarity. Water Resour. Res. 17(2), 337-350 (1981)

Haberman, R.: Elementary Applied Partial Differential Equations: With Fourier Series and Boundary Value. Prentice Hall, Upper Saddle River (1998)

Li, H., Zhang, D.: Probabilistic collocation method for flow in porous media: comparisons with other stochastic methods. Water Resour. Res. 43, W09409 (2007)

Li, L., Graham, W.D.: Stochastic analysis of solute transport in heterogeneous aquifers subject to spatiotemporal random recharge. Water Resour. Res. 35(4), 953-971 (1999)

Li, S.-G., McLaughlin, D.: A nonstationary spectral method for solving stochastic groundwater problems: Unconditional analysis. Water Resour. Res. 27(7), 1589-1605 (1991)

Li, W., Lu, Z., Zhang, D.: Stochastic analysis of unsaturated flow with probabilistic collocation method. Water Resour. Res. 45, W08425 (2009)

Liu, G., Lu, Z., Zhang, D.: Stochastic uncertainty analysis for solute transport equation approach. Water Resour. Res. 43, W07427 (2007)

Lu, Z., Zhang, D.: Stochastic analysis of transient flow in heterogeneous, variably saturated porous media: The van Genuchten-Mualem constitutive model. Vadose Zone Jurnal 1, 137-149 (2002)

Mantoglou, A., Gelhar, L.W.: Stochastic modeling of large-scale transient unsaturated flow systems. Water Resour. Res. 23(1), 37-46 (1987)

Mantoglou, A.: A theoretical approach for modeling unsaturated flow in spatially variable soils: effective flow models in finite domains and nonstationarity. Water Resour. Res. 28(1), 251-267 (1992)

Rodriguez-Iturbe, I., Isham, V., Cox, D.R., Manfreda, S., Porporato, A.: Space-time modeling of soil moisture : stochastic rainfall forcing with heterogeneous vegetation. Water Resour. Res 42, W06D05 (2006)

Rubin, Y.: Applied Stochastic Hydrogeology. Oxford University Press, New York (2003)

Rupp, D.E., Keim, R.F., Oissander, M., Brugnach, M.F., Selker, J.S.: Time scale and intensity dependency in multiplicative cascades for temporal rainfall disaggregation. Water Resour. Res. 45, W07409 (2009)

Russo, D.: On the velocity covariance and transport modeling in heterogeneous anisotropic porous formations 2 Unsaturated flow. Water Resour. Res. 31(1), 139-145 (1995)

Settin, T., Botte, G., Rodriguez-Iturbe, I., Rinaldo, A.: Numerical studies on soil moisture distributions in heterogeneous catchments. Water Resour. Res. 43, W05425 (2007)

Veneziano, D., Iacobellis, V.: Multiscaling pulse representation of temporal rainfall. Water Resour. Res. 38(8), 1138 (2002)

Yoo, C., Valdés, J.B., North, G.R.: Valuation of the impact of rainfall on soil moisture variability. Adv. Water Resour. 21(5), 375-384 (1998)

Zhang, D.: Stochastic Methods for Flow in Porous Media: Coping with Uncertainties. Academic Press, San Diego (2002)

Zhang, D., Lu, Z.: An efficient, high-order perturbation approach for flow in random porous media via Karhunen-Loe've and polynomial expansions. J. Comput. Phys. 194, 773-794 (2004)

Zhang, D., Lu, Z.: Stochastic analysis of flow in a heterogeneous unsaturated-saturated system. Water Resour. Res. 38(2), 1018 (2002). doi:10.1029/2001WR000515

Zhang, D., Winter, C.L.: Moment equation approach to single phase fluid flow in heterogeneous reservoirs. Soc. Petrol Eng. J. 4(2), 118-127 (1999)

Zhang, Y.-K., Li, Z.: Effect of temporally correlated recharge on fluctuations of groundwater levels. Water Resour. Res. 42(10), W10412 (2006). doi:10.1029/2005WR004828 